\title{
Tolerability of antipsychotic drugs: does patient perspective deserve consideration?
}

\author{
MICHELA NOSÈ
}

\begin{abstract}
This contribution initially describes some traditional tools that are commonly used to measure drug tolerability, including measures that take into considerations both clinicians' and patients' views. Subsequently, it highlights a few studies that compared the patient and clinician's perspective in the evaluation of drug tolerability, trying to understand whether health care providers and patients perceive antipsychotic tolerability in different ways, and whether these different ways may have implications in terms of treatment adherence and outcome. Finally, some clinical and research implications are suggested and discussed.
\end{abstract}

There is overwhelming evidence that antipsychotic drugs are effective in both acute treatment and relapse prevention of schizophrenia and related disorders. Despite this, antipsychotics are associated with a wide range of side effects and, for a number of patients, these adverse reactions can cause even greater level of distress than the symptoms of the illness (Morrison et al., 2000). Many studies have shown that these factors affect patients in several ways with a possible impact on different areas, including physical morbidity and stigma, quality of life and treatment adherence (Hamer \& Haddad, 2007). In community psychiatric settings, treatment non-adherence remains a crucial problem that can result in long-term therapeutic plans being ineffective (Nosè et al., 2003a). In clinical practice, in patients treated with antipsychotics, non-adherence may have wider clinical implications. Poor compliance with antipsychotic medications, for example, is associated with increased psychotic relapse, hospital admissions and poorer outcomes (Day et al., 2005; Gray et al., 2002); it may additionally cause problems in the management of firstepisode schizophrenic patients (McGorry \& Killackey, 2002). Generally, adherence is considered a multi-determined phenomenon with many different factors associated with it, but side-effects and attitude towards medications seem to strongly influence this phenomenon (Nosè et al., 2003b; Kampman et al., 2002; Fenton et al., 1997).

Address for correspondence: Dr. M. Nosè, Department of Medicine and Public Health, Section of Psychiatry and Clinical Psychology, University of Verona, Piazzale L.A. Scuro 10, 37134 Verona (Italy).

Fax: +39-045-585871

E-mail: michela.nose@univr.it

Declaration of Interest: None.
Although antipsychotics are associated with a very wide range of side-effects, and side-effects play a crucial role in treatment adherence according to how they are perceived by patients, epidemiological studies and clinical trials have rarely taken into consideration these aspects so far. Typically, clinical and epidemiological studies have described extrapyramidal side effects (EPS) only, but patients may report a wider range of adverse reactions. In addition, the recent introduction of novel or "atypical" antipsychotic agents has further complicated this picture, considering that novel antipsychotic are less associated with EPS but are more likely to cause metabolic side effects. Recognizing and taking action on all kind of adverse reactions need to be seen as an important aspect of medication management for all drugs, but it is now clear that the health care provider viewpoint is not the only perspective that has to be considered. In the recent literature the patients' perspective on drug tolerability has become one of the most important elements in the evaluation of side effects and, particularly, in the evaluation of the impact that these may have on patient's adherence, quality of life and long-term outcome.

In this editorial, I will initially describe the traditional tools that are used to measure drug tolerability, trying to include measures that take into considerations both clinicians' and patients' views. Subsequently, I will highlight a few studies that compared patient and clinician's perspective in the evaluation of drug tolerability, trying to understand whether health care providers and patients perceive antipsychotic adverse effects in different ways, and whether these different ways may have implications in terms of treatment adherence and outcome. Finally, some clinical and research implications will be suggested and discussed. 


\section{MEASURING TOLERABILITY}

There are a few scales commonly used for the routine clinical measurement of a broad array of antipsychotic side effects. The first instruments for formalizing antipsychotic adverse reactions were developed by neurologists in the USA with the aim of assessing the novel forms of parkinsonism and tardive diskynesias that emerged in the 1960s, with the introduction of antipsychotics in clinical practice. Subsequently, these have been developed into scales for assessing specific posture and movement disorders, for example the Barnes scale (Barnes, 1989), the Simpson \& Angus Scale (Simpson \& Angus, 1970), and the DIES (Drug Induced Extrapyramidal Disorder) scale (Inada et al., 2003). Another well known instrument for measuring antipsychotic side-effects is the UKU (Udvalg for Kliniske Unedrsolgelser) Side Effects Rating Scale (Lingjaerde et al., 1987). The UKU has been shown to have good psychometric properties. Using a semi-structured interview, this instrument measures 48 individual side effects. It was formulated for use in different psychiatric conditions ranging from psychosis to affective and neurotic disorders. All these scales measure a very limited range of adverse effects, as perceived by health care providers. More recently, from the UKU scale a self-report measure for adverse effects related to antipsychotic treatment has been developed: the Liverpool University Neuroleptic Side Effects Rating Scale (LUNSERS) (Day et al., 1995). The LUNSERS comprises 51 items, 41 of which are modelled as closely as possible on corresponding items in the UKU. Despite some initial concern and scepticism regarding the possibility of people with mental disorders to credibly assess their mental or physical state, the LUNSERS has been shown to have a good test reliability and concurrent validity against the UKU (Lambert et al., 2003). Clearly, the use of a self-reported scale is particularly important in the evaluation of antipsychotic tolerability, considering that the patient's subjective experience has a key role in determining the success or failure of any drug treatment.

\section{PATIENT'S PERSPECTIVE}

Unfortunately, over the last few decades the patients' view has received very little attention and research on adverse effects has largely been concerned with quantifying symptoms rather than determining their impact on patients. However, in recent years there has been an increasing interest in the subjective view of patients, including their views on antipsychotic medication regimens (Hamer \& Haddad, 2007; Voruganti et al., 2000). Data in the literature are hardly comparable, because dif- ferent designs were adopted, but generally the most frequent antipsychotic adverse reactions reported by patients were weight gain, tiredness, difficulty remembering and concentrating and sleeping too much (Morrison et al., 2000; Voruganti et al., 2000). Castle et al. (2002), who assessed the patients' perspective towards antipsychotic drugs in a sample of 998 patients with psychotic disorders treated in Australia, showed that nearly 80\% reported at least one side-effect. Moreover, many side-effects may go unnoticed because patients may not report them and staff do not ask about possible problems that patients may experience. On the other hand, in some cases patients do report specific side effects, but these may be underestimated as a possible cause of distress and, consequently, their impact on adherence is neglected.

It has been noted that many variables may influence the patient's perspective of tolerability, including socio-demographic characteristics. Gender differences, for example, have sometimes been noticed. In a study that recruited patients with schizophrenia under the care of psychiatric services serving geographical catchment areas in Croydon (UK), Verona (Italy), Amsterdam (Netherlands), and Leipzig (Germany), we rated antipsychotic subjective tolerability using the LUNSERS (Barbui et al., 2005). During the recruitment period, 245 men and 164 women with schizophrenia were recruited. In both sexes the most frequently reported side-effects were difficulty in concentrating, tiredness and weight gain; these side-effects occurred in around $50 \%$ of men and in up to $70 \%$ of women. Extrapyramidal and anticholinergic reactions were reported more often by women, while men reported sexual problems more often. After background group differences were controlled for, gender was the strongest determinant of the subjective tolerability of antipsychotic drugs. This gender difference may have implications for practice and for research. For example, it is well recognized that the process of making a decision on which drug should be prescribed in each single patient requires careful consideration of many different aspects and, among these aspects, gender should probably be included when antipsychotic drugs are prescribed. The finding that women do not perceive antipsychotic drugs less tolerable than men in general, but only with respect to some specific side-effects, and the fact that there are differences between antipsychotic drugs in the occurrence of specific side-effects, could help guide the choice of antipsychotic drugs. For example, women perceive extrapyramidal symptoms as more troublesome than the men, and this could counterbalance other reasons for prescribing conventional drugs - good response in the past, for example. Nevertheless, novel drugs differ with respect to the incidence of weight gain, negatively perceived more often by women than man. 
These considerations should routinely be taken into consideration and discussed with patients, careers and family members when deciding the most appropriate drug to prescribe. Additionally, in patients already in treatment, physicians should consider these gender differences, at least in two ways:

a) by recognizing that women might be stabilized at slightly lower doses than men, decreasing this way the perceived burden of side-effects;

b) by systematically investigating patients' perceptions of side-effects in a focused way, for example exploring men' perception of sexual problems and women's perception of weight gain. Obviously, we still don't know whether these physicians' attitudes might have a positive impact on patients' perception and, perhaps, treatment adherence and outcome.

\section{CLINICIANS' AND PATIENTS' PERSPECTIVE AS TWO COMPLEMENTARY DIMENSIONS IN THE EVALUATION OF ANTIPSYCHOTIC DRUGS}

Do health care professionals and patients have similar concerns about antipsychotic side effect? Is the clinician's perspective overlapping with the patient's perspective?

Finn et al. (1990), who carried out a study that measured the subjective burden of both psychotic symptoms and medication side effects in patients and clinicians, found that the side effects of antipsychotics were perceived as being as troublesome as the symptoms they were being used to treat by patients; psychiatrists, by contrast, considered side effects less bothersome than symptoms. Moreover, a substantial disagreement between patients' and psychiatrists' ratings of troublesomeness of specific side effects was found. These observations raise the possibility that prescribers may not always be accurate in understanding the influence that side effects may have on the lives of patients.

Day et al. (1998) noted that patients and psychiatrists share similar views about the prevalence and implications of antipsychotic side-effects. However, psychiatrists had not idea of which side-effects were most likely to cause distress to patients, and this was mentioned as a possible reason for adversely affecting the therapeutic alliance. In general, psychiatrists were aware of the level of discomfort experienced by patients as a consequence of taking antipsychotic medication, but the correlation between the psychiatrists' ratings of distress and those made by the patients was poor, indicating that psychiatrists were unaware of which particular side effects were more distressing. Huffman et al. (2004), who compared the clinician and patient concerns about five antipsychotic side effects, found that patients may be more concerned than clinicians about cognitive slowing. Cognitive slowing is somewhat more subjective, less obvious and less medically dangerous than other side effects, but it may heavily impair a person's daily function, and, consequently, may have a negative impact on treatment adherence.

In summary, although findings from the literature are still very limited, it is clear that, in addition to clinicians' view, patients' view cannot be ignored any longer.

\section{CLINICAL IMPLICATIONS}

There is an urgent need to better assess and monitor antipsychotic side effects in clinical practice, and staff needs to be properly trained in this assessment and monitoring. This may encourage staff to keep patients better informed about side effects and it may additionally encourage patients to communicate every side effect to staff members. In clinical practice, patients should be informed of common side-effects prior to treatment and should additionally be regularly monitored during treatment (Hamer \& Haddad, 2007). Routine assessment of side effects should be an integral part of clinical practice for patients taking antipsychotics, and doctors should be more open to discussing the risks and nature of adverse effects with their patients.

Many prescribers are reluctant to provide patients with information about side effects because this might reduce adherence. However, data from the literature indicate that warning patient about severe side effects does not have a negative impact on adherence; if anything, the contrary may be true, that is warning may reduce anxiety about taking medicines (Day et al., 1998). In addition, clinicians sometimes believe that it is not necessary to warn patients about possible risks because this might induce over-reporting. Even this belief is not supported by any evidence.

\section{RESEARCH IMPLICATIONS}

From an epidemiological viewpoint, studies describing the tolerability of antipsychotics in terms of views expressed by patients and clinicians are urgently needed. The possibility of integrating different points of view may represent a way to develop a better therapeutic alliance that might decrease the likelihood of non-adherence. Moreover, it is clear that, in addition to a quantification of the prevalence of side effects, their impact on everyday life needs to be better described and quantified. In SouthVerona, an observational study aimed to describe patient, clinician and key-operator's beliefs about antipsychotic tolerability is currently under way. The prevalence and

Epidemiologia e Psichiatria Sociale, 17, 3, 2008 
impact of side effects for a consecutive case-series of patients receiving antipsychotics is measured, and the different perspectives are compared trying to highlight differences and check whether patient and clinician ratings are influenced by similar or different factors. For each reported adverse reaction patients are asked to estimate the level of distress that is experienced. Psychiatrists and key operators are asked to complete a similar questionnaire.

In addition to epidemiological surveys, another key research implication refers to the need of incorporating measures of subjective tolerability as outcome parameters in clinical trials. In recent years, tolerability and acceptability has been considered a key aspect in the famous CATIE study (Liebermann et al., 2005), where treatment discontinuation was employed as the primary outcome measure. Authors argued that the proportion of patients discontinuing treatment may provide a useful global outcome measure that simultaneously incorporates tolerability and efficacy, and simultaneously incorporates patient and clinician inputs. In Italy, a clinical trial (Barbui et al., 2006) has recently been launched to test the relative efficacy and tolerability of combination treatment with clozapine plus aripiprazole compared to combination treatment with clozapine plus haloperidol in patients with an incomplete response to clozapine alone. In this study the primary outcome is treatment discontinuation. Again, it has been argued that this measure may represent a pragmatic endpoint that reflects both clinician and patient judgments about efficacy and tolerability. The recently published EUFEST trial employed a similar primary outcome in a very different patient population exposed to antipsychotic drugs (Kahn et al., 2008).

Hopefully, in the near future clinical trials will increasingly include measures of subjective tolerability as clinically sound outcomes. Researchers and health care providers interested in increasing treatment adherence and patient outcomes should increasingly consider clinicians' and patients' perspective as two complementary dimensions that provide key insights in the evaluation of antipsychotic drugs.

\section{REFERENCES}

Barbui C., Nosè M., Bindman J., Schene A., Becker T., Mazzi M.A., Kikkert M., Camara J., Born A. \& Tansella M. (2005). Sex differences in the subjective tolerability of antipsychotic drugs. Journal of Clinical Psychopharmacology 25, 521-526.

Barbui C., Cipriani A., Malvini L., Nosè M., Accordini S., Pontarollo F., Veronese A., Tansella M \& CHAT investigators (2006). Trasformare la pratica clinica in ricerca. Un invito a partecipare allo studio CHAT. Rivista di Psichiatria 41, 326-330.

Barnes T.R.E. (1989). A rating scale for drug-induced akathisia. British Journal of Psychiatry 154, 672-676.

Castle D., Morgan V. \& Jablensky A. (2002). Antipsychotic use in Australia: the patients' perspective. Australian \& New Zealand Journal of Psychiatry 36(5), 633-641.

Day J.C., Wood G., Dewey M. \& Bentall R.P. (1995). A self-rating scale for measuring neuroleptic side-effects. Validation in a group of schizophrenic patients. British Journal of Psychiatry 166, 650-653.

Day J.C., Kinderman P. \& Bentall R. (1998). A comparison of patients' and prescribers' beliefs about neuroleptic side-effects: prevalence, distress and causation. Acta Psychiatrica Scandinavica 97(1), 93-97.

Day J.C., Bentall R.P., Roberts C., Randall F., Rogers A., Cattell D., Healy D., Rae P. \& Power C. (2005). Attitudes toward antipsychotic medication. Archives of General Psychiatry 62, 717-724.

Fenton W.S., Blyler C.R. \& Heinssen R.K. (1997). Determinants of medication compliance in schizophrenia: empirical and clinical findings. Schizophrenia Bulletin 23, 637-651.

Finn S.E., Bailey J.M., Schultz R.T. \& Faber R. (1990). Subjective utility ratings of neuroleptic in treating schizophrenia. Psychological Medicine 20, 843-848.

Gray R., Robson D. \& Bressington D. (2002). Medication management for people with a diagnosis of schizophrenia. Nursing Time 98, 38-40.

Hamer S. \& Haddad P. (2007). Adverse effects of antipsychotics as outcome measures. British Journal of Psichiatry 191, Suppl 50, 64-70.

Huffman J.C., Blais M.A. \& Pirl W.F. (2004). A comparison of clinician and patient concerns about antipsychotic side effects: a pilot study. Journal of Nervous and Mental Disorder 192, 328-330.

Inada T., Beasley C.M., Tanaka Y. \& Walker D.J. (2003). Extrapyramidal symptom profiles assessed with the Drug-Induced Extrapyramidal Symptom Scale. International Clinical Psychopharmacology 18, 39-48.

Kahn R.S., Fleischhacker W.W., Boter H., Davidson M., Vergouwe Y., Keet I.P., Gheorghe M.D., Rybakowski J.K., Galderisi S., Libiger J., Hummer M., Dollfus S., López-Ibor J.J., Hranov L.G., Gaebel W., Peuskens J., Lindefors N., Riecher-Rössler A., Grobbee D.E. \& EUFEST study group (2008). Effectiveness of antipsychotic drugs in first-episode schizophrenia and schizophreniform disorder: an open randomised clinical trial. Lancet 371, 1085-97.

Kampman O., Laippala P., Vaananen J., Koivisto E., Kiviniemi P., Kilkku N. \& Lehtinen K. (2002). Indicators of medication compliance in first episode psychosis. Psychiatry Research 110, 39-48.

Lambert T.J.R., Cock N., Alcock J., Kelly D.L. \& Conley R.R. (2003). Measurement of antipsychotic-induced side effects: support for the validity of self-report (LUNSERS) versus structured interview (UKU) approach to measurement. Human Psychopharmacoly-Clinical and Experimental 18, 405-411.

Lieberman J.A., Stroup T.S., McEvoy J.P., Seartz M.S., Rosenheck R.A., Perkins D.O., Keefe R.S., Davis C.E., Lebowitz B.D., Severe J., Hsiao J.K. \& Clinical Antipsychotic Trials of Intervention Effectiveness (CATIE) Investigators. (2005). Effectiveness of antipsychotic drugs in patients with chronic schizophrenia. New England Journal of Medicine 353, 1209-1223.

Lingjaerde O., Ahlfors V.G. \& Dech P. (1987). The UKU side effect rating scale for psychotropic drugs and a cross sectional study of side effects in neuroleptic treated patients. Acta Psychiatrica Scandinavica, Supplementum No. 334, 76.

McGorry P.D. \&, Killackey E.J. (2002). Early intervention in psychosis: a new evidence based paradigm. Epidemiologia e Psichiatria Sociale 11, 237-247.

Morrison P., Gaskill D., Meehan T., Lunney P., Lawrence G. \& Collings P. (2000). The use of the Liverpool University Neuroleptic Side-Effect Rating Scale (LUNSERS) in clinical practice. Australian \& New Zealand Journal of Mental Health Nursing 9, 166-176.

Nosè M., Barbui C., Gray R. \& Tansella M. (2003a). Clinical interventions for treatment non-adherence in psychosis: meta-analysis. British Journal of Psychiatry 183, 197-206.

Nosè M., Barbui C. \& Tansella M. (2003b). How often do patients with psychosis fail to adhere to treatment programmes? A systematic review. Psychological Medicine 33, 1149-1160.

Simpson J.A. \& Angus J.W.S (1970). A rating scale for extrapyramidal side effects. Acta Psychiatrica Scandinavica, Supplementum 212, 11-19.

Vorunganti L., Cortese L., Oyewumi L., Cernovsky Z., Zirul S. \& Awad A. (2000). Comparative evaluation of conventional and novel antipsychotic drugs with reference to their subjective tolerability, side-effect profile and impact on quality of life. Schizophrenia Research 43, 135-145. 